\title{
Nueva gestión pública y reforma educativa: La recontextualización de la autonomía escolar en diferentes contextos escolares en Cataluña ${ }^{1}$
}

\section{Lluís Parcerisa ${ }^{\mathrm{i}}$}

Universitat Autònoma de Barcelona, España

Resumen

La Nueva Gestión Pública (NGP) emergió en el debate político a partir de la década de los ochenta, bajo la promesa de mejorar la efectividad del sector público, mediante el modus operandi de la empresa privada. La autonomía escolar es una política clave en la agenda de la NGP. Esta investigación analiza la recontextualización de la autonomía escolar en la escuela pública en Cataluña. La hipótesis principal es que la composición social y la estabilidad de la plantilla docente influyen en la recontextualización de la autonomía escolar. La metodología utilizada se basa en el estudio de caso de tipo comparativo. Dos escuelas públicas con diferentes contextos escolares han sido seleccionadas. El estudio de caso combina encuestas con la plantilla docente, entrevistas semiestructuradas con actores clave y observación directa. Los resultados muestran que el contexto escolar puede desencadenar respuestas diferenciales y limitar el desarrollo de la autonomía pedagógica en contextos desaventajados.

Palabras-clave

Nueva Gestión Pública; Autonomía escolar; Recontextualización; Privatización endógena 


\section{Introducción}

En los últimos tiempos se ha producido la expansión de un verdadero movimiento de reforma educativa a escala global, el cual está produciendo cambios importantes en la configuración de los sistemas educativos de todo el mundo (Sahlberg, 2006). A través de este movimiento de reforma educativa se está promoviendo la introducción de las ideas y las técnicas gerenciales que provienen del sector privado, en los sistemas educativos públicos (Verger, 2012), con el objetivo de resolver los principales problemas de la educación (Verger \& Altinyelken, 2013).

Estas medidas a menudo se relacionan con la Nueva Gestión Pública (NGP), la cual ha tenido un peso importante en las reformas educativas que se han llevado a cabo en los países del sur de Europa (véase Grimaldi \& Serpieri, 2013; Landri, 2009; Luengo \& Saura, 2012; Merchán, 2012; Verger \& Curran, 2014). Sin embargo, las motivaciones que se esconden detrás de su adopción en cada país o región son muy diversas. En este sentido, lejos de basarse en un esquema de reforma monolítico, bajo el paraguas de la NGP podemos encontrar políticas con diseños y lógicas muy diferentes (Verger \& Normand, 2015).

En el marco del estado español, la comunidad autónoma de Cataluña, junto con la de Andalucía, fue pionera en la promoción e implementación de la NGP en las escuelas públicas (Merchán, 2012; Saura, 2015). Posteriormente, la NGP también tendría un papel central en la controvertida reforma LOMCE (Ley Orgánica 8/2013, de 9 de diciembre), que fue promulgada por el Partido Popular. En este sentido, si bien es cierto que en los últimos años la introducción de la NGP ha generado un importante debate a nivel político, académico y social, lo cierto es que aún disponemos de pocas evidencias para poder hacer una valoración fundamentada científicamente alrededor del funcionamiento y los efectos de dicha política, y, lo que es más relevante, si ésta tiene éxito y en qué contextos.

Una de las medidas políticas más relevantes dentro de la agenda de la NGP es, sin lugar a dudas, la autonomía escolar. Organismos internacionales como la OCDE, a través del prestigioso informe PISA, han contribuido a diseminar las políticas de autonomía escolar en todo el mundo, presentándolas como una buena práctica para la mejora del rendimiento 
(Bonal \& Verger, 2013a) y para la reducción del abandono escolar prematuro (Garcia \& del Campo, 2012), que es una de las problemáticas más relevantes a nivel europeo, y particularmente de España (García, 2005; Tarabini, Curran, Montes, \& Parcerisa, 2015). No obstante, el concepto de autonomía escolar puede servir para justificar políticas con diferentes enfoques. Es por ello que resulta importante ahondar en el estudio de la autonomía escolar, con el fin de abrir la caja negra y comprender qué teoría del cambio hay detrás y cómo es reinterpretada y traducida por los actores encargados de su aplicación, en diferentes contextos.

Mediante la presente investigación se pretende analizar la recontextualización de las ideas de autonomía escolar subyacentes a la Ley de Educación de Cataluña (LEC), y los distintos decretos que la desarrollan, en las escuelas públicas catalanas. La investigación explora las consecuencias que genera su aplicación en diferentes contextos escolares y cuáles son las dimensiones de la autonomía que toman más relevancia en este proceso.

\section{La Nueva Gestión Pública (NGP) como mecanismo de privatización endógena de la educación}

La NGP se ha empleado en el discurso de la reforma de la administración pública por motivaciones diversas, y su implantación ha sido defendida por partidos políticos situados en posiciones de la derecha y de la izquierda del espectro político, indistintamente (Verger \& Curran, 2014; véase también Saura, 2015). El término NGP se empezó a emplear en la década de 1980 (Hood, 1991; Sehested, 2002) en países como EEUU, Canadá o Reino Unido y se vincula con la realización de una verdadera revolución en la gestión de la administración pública, donde se quieren introducir los conocimiento y las técnicas procedentes del ámbito empresarial, para favorecer una mejora en el rendimiento de los servicios públicos, aumentando su eficiencia y eficacia (Common, 1998). Los defensores de la NGP consideran que la excesiva rigidez y burocratización del sector público hacen que este sea ineficiente (Barzelay, 2001). En consecuencia, la NGP es una filosofía que busca la desburocratización de los servicios públicos para hacerlos más efectivos (Verger, 2007). 
Aunque puede parecer contra-intuitivo, la NGP no supone la desaparición del Estado. Más bien, lo que se produce es una transformación de sus funciones clásicas, de tal manera que el Estado aumenta sus funciones regulatorias y su rol vigilante mediante la aplicación de evaluaciones externas, al mismo tiempo que se encarga de distribuir incentivos entre los distintos actores que operan en el campo escolar para estimular la mejora del rendimiento (Verger \& Curran, 2014). En este sentido, cabe destacar que a menudo la NGP va de la mano del concepto accountability (o rendición de cuentas) y entre ambos dan lugar a lo que se conoce como "el Estado evaluador" (Neave, 1998; Hartley, 2001).

En el contexto de la reforma de la administración pública, sectores como la educación y la sanidad se han visto muy afectados por las reformas gerenciales de la NGP. En el caso de la educación, estas reformas han producido cambios en la gobernanza de las instituciones educativas (Tolofari, 2005). Algunos autores consideran la NGP como una forma de privatización (véase Ball \& Youdell, 2007).

Cuando hablamos de privatización, a menudo se hace la distinción entre las formas de privatización de la educación y las formas de privatización en la educación. La NGP generalmente se define como una forma de "privatización endógena" (Ball \& Youdell, 2007; Ball, 2010). Los defensores de estas formas de privatización parten de la premisa de que el sector privado acostumbra a funcionar más eficientemente que el sector público. Así pues, a través de este enfoque de privatización endógena, lo que se pretende es incorporar en la escuela pública la arquitectura institucional, los métodos de trabajo y las prácticas que se aplican en el mundo empresarial (Bernal, Cano, \& Lorenzo, 2014).

En el ámbito educativo, la NGP se traduce en la incorporación de sistemas de evaluación estandarizados, la publicación de tablas de resultados que fomentan la competición entre centros, la autonomía institucional de las unidades (en este caso de las escuelas), el fomento de la libre elección de centro, la profesionalización y la concentración de poder en la dirección, en detrimento de los órganos colegiados (Ball \& Youdell, 2007; Bernal \& Lorenzo, 2012; Tolofari, 2005). En definitiva, la NGP se introdujo en el campo educativo bajo la promesa de mejorar los resultados y la calidad educativa y aumentar la competitividad (Wittmann, 2008). 
Dentro del paquete de reformas de la NGP en educación, una de las políticas más populares es la autonomía escolar, que a menudo se acompaña con medidas de rendición de cuentas. A continuación se presentan algunos de los enfoques que pueden tomar las políticas de autonomía escolar y posteriormente se describe brevemente el proceso de adopción y el desarrollo normativo de la autonomía escolar en Cataluña.

\section{NGP y autonomía escolar: definición de un concepto polisémico}

Generalmente la autonomía escolar se define como la descentralización de las responsabilidades y el poder en la toma de decisiones, que se transfieren a las escuelas (Espínola, 2000). Sin embargo, autores como Barroso (2004) consideran que detrás de la autonomía escolar se esconde una estrategia de reforma de la organización de los centros educativos.

Según Calero (2010), la idea de autonomía escolar puede derivar en políticas y en prácticas que pueden tener diferentes impactos sobre calidad y la equidad educativa. En esta línea, algunos autores señalan que la autonomía escolar es un "significante flotante" que se acomoda fácilmente a diferentes narrativas sobre la reforma educativa, de manera que es aceptada por audiencias con ideologías muy dispares (Verger \& Normand, 2015).

Por lo tanto, en la práctica el concepto de autonomía escolar funciona como un concepto paraguas bajo el cual se esconden diseños, medidas y lógicas que varían sustancialmente. Por ejemplo, los autores adscritos al neoliberalismo perciben la autonomía escolar como un mecanismo adecuado para reducir el rol del Estado y mercantilizar el sector público, incorporando la lógica de funcionamiento del sector privado en las escuelas públicas; para los conservadores, la autonomía escolar es una buena manera de profesionalizar la dirección escolar, aumentando su rol gerencial y delegándole responsabilidades y poder en la toma de decisiones. De esta manera, se jerarquiza la gobernanza y la gestión de las escuelas; en cambio, los socialdemócratas perciben la autonomía escolar como una manera de aumentar la eficacia y la eficiencia del sector público. En consecuencia, la mejora de la efectividad permite defender a los servicios públicos frente a los 
promotores de la privatización, que los acusan de ser excesivamente rígidos, burocráticos y poco eficientes; por último, para los docentes que participan en movimientos de renovación pedagógica la autonomía se considera una política idónea para impulsar la innovación pedagógica en los centros y favorecer una educación más contextualizada (Verger, Curran, \& Parcerisa, 2015).

A nivel académico se identifican diferentes modelos de autonomía escolar. Por ejemplo, Meuret (2004) identifica tres tipos ideales: el modelo administrativo, el profesional y el comunitario, los cuales se distinguen por entregar el poder en la toma de decisiones a la dirección escolar, los docentes y a la comunidad, respectivamente.

Por otro lado, Estruch (2006) distingue entre la autonomía neoliberal, la autonomía corporativista y la autonomía integradora. La autonomía neoliberal opera en sistemas educativos regidos por la lógica de mercado, que otorgan el poder a las familias (definidas como "consumidores") mediante la libre elección de centro. Sin embargo, la transferencia de poder a las familias no socava el poder del Estado, sino que éste ve como se transforman sus funciones, convirtiéndose en un Estado evaluador (Neave, 1998). En la autonomía corporativista el poder de decisión recae en los profesionales que trabajan en el centro educativo.

Por último, la autonomía integradora nace como una crítica a los dos modelos anteriores. La principal diferencia respecto el modelo neoliberal es la exclusión del principio de competencia entre escuelas. Por otro lado, también critica los efectos perversos del poder corporativo y defiende un modelo similar al finlandés, donde los centros contratan directamente a los docentes, que no son funcionarios pero tienen un elevado prestigio social y trabajan de manera colegial para lograr los objetivos fijados a nivel municipal en un marco de liderazgo pedagógico (Estruch, 2006).

Una variable que aparece estrechamente vinculada a la autonomía escolar es el liderazgo (Pont, Nusche, \& Moorman, 2009). De hecho, según autores como Garcia y del Campo (2012), la autonomía escolar debe ir acompañada de un modelo de liderazgo pedagógico y distribuido, el cual se considera un requisito indispensable para el buen funcionamiento de la autonomía, así como para mejorar la eficacia y la promoción de la innovación. A su vez, una extensa literatura identifica diferentes clasificaciones y modelos 
de liderazgo, y se ocupa de su relación con la autonomía escolar (véase Barroso, 2005; Bolívar, 2001; Gunter, 2001; Larruzea, 2013; Pont et al., 2009; Spillane, Halverson, \& Diamond, 2001; OCDE, 2009).

En cuanto a la evidencia internacional, la OCDE señala que en los países donde hay más escuelas que disfrutan de autonomía pedagógica, el rendimiento de todo el sistema escolar es mejor. En contraste, se observa que no existe una relación causal entre la autonomía de gestión y el rendimiento académico que se consigue a nivel estatal (OCDE, 2011). Por otro lado, en relación con la autonomía institucional de las escuelas también se afirma que "los datos PISA muestran que la relación entre el rendimiento de los centros educativos individuales y su nivel de autonomía en la asignación de recursos es positiva en algunos países y negativa en otros" (OCDE, 2011, p. 2).

A pesar de que la evidencia internacional es poco concluyente a la hora de demostrar las mejoras que genera la autonomía escolar y que a menudo se acostumbra a jugar con la ambigüedad de este concepto para legitimar políticas con orientaciones ideológicas antagónicas, lo cierto es que los informes de la OCDE, y los de PISA en particular, se acostumbran a emplear en el debate político para legitimar opciones políticas particulares en la reforma educativa (Bonal \& Tarabini, 2013).

\section{Adopción y desarrollo de las ideas de la NGP en el sistema educativo catalán}

A nivel estatal, Cataluña es una de las comunidades autónomas con una regulación más desarrollada en el ámbito de la autonomía escolar (Sancho, 2014). En este sentido, la OCDE fue clave en el proceso de construcción de la autonomía como preferencia política de los policy-makers locales.

En Cataluña la OCDE, a través de PISA y otros estudios, facilitó a los policy-makers locales la identificación de problemas y las posibles soluciones en materia de política educativa. Además, también fue una de las principales fuentes de inspiración para los policy-makers catalanes, que adoptaron algunas de sus ideas sobre el liderazgo escolar y la autonomía de centro. Sin embargo, la interpretación que hicieron de los datos y recomendaciones procedentes de la OCDE en muchas ocasiones fue parcial o sesgada (Verger et al., 2015). 
En Cataluña la NGP fue adoptada por un Gobierno socialdemócrata formado por una coalición integrada por socialistas (PSC), independentistas socialdemócratas (ERC) y eco-socialistas (ICV-EUiA) (Verger et al., 2015). La coalición socialdemócrata gobernó durante dos legislaturas (2003-2006 y 2006-2010). Durante la primera legislatura se elaboró un Acuerdo Nacional de Educación (Maragall \& Colomé, 2013). Además, entre los años 2004 y 2005 el Departamento de Educación configuró el diseño de un Plan de Autonomía de Centro (Plan PAC) que pretendía introducir cambios profundos en el modelo de gestión de la organización escolar (Garcia \& del Campo, 2012) y avanzar hacia un modelo basado en la idea de corresponsabilidad (ENINSDEPEDUC092; Garcia \& del Campo, 2012).

En esta primera fase el programa de pilotaje de la autonomía que se aplicó adoptó un enfoque pedagógico. Sin embargo, en la segunda legislatura fue cuando se produjo una verdadera transformación, mediante la introducción de un enfoque gerencial de la NGP en el marco regulatorio, que se concretó con la aprobación de la Ley de Educación de Cataluña (LEC) en el año 2009. Durante la segunda legislatura también se promulgaron los principales decretos mediante los cuales se pretendía desarrollar la LEC, aunque no todos se pudieron hacer efectivos. Esta ley incorporaba un enfoque gerencial de la autonomía y otorgaba competencias y poder en la toma de decisiones a la dirección escolar, y al mismo tiempo socavaba el poder de los docentes. Posteriormente, durante el gobierno conservador (2010-hasta la actualidad) la NGP fue implementada de manera selectiva por parte del Gobierno conservador (Verger et al., 2015).

A nivel normativo la LEC cuenta con tres pilares, a saber: la autonomía de centro, la evaluación y la dirección escolar (Bonal \& Verger, 2013b), que se desarrollan a través de diferentes decretos, como son el decreto de autonomía escolar (Decreto 102/2010, 3 de agosto de 2010), el decreto de dirección (Decreto 155/2010, 2 de noviembre de 2010), el decreto de evaluación (Decreto 177/2010, 23 de noviembre de 2010) y, por último, el controvertido decreto de provisión de plantillas (Decreto 39/2014, de 25 de marzo de 2014). Sin embargo, en el caso del decreto de evaluación, el Gobierno conservador suspendió la creación de la agencia de evaluación debido a motivaciones de carácter económico e ideológico (véase Verger et al., 2015). 


\section{La recontextualización de las políticas educativas a nivel local}

Generalmente, tanto la literatura académica como los gobiernos se han referido al proceso de implementación de políticas públicas, asumiendo que se trata de un proceso lineal (top-down o bottom-up), sin resistencias (Thrupp \& Easter, 2012). Sin embargo, las políticas educativas deben aplicarse en contextos locales reales, es decir, en escuelas que operan dentro de un espacio educativo local particular. Además, generalmente estas disponen de recursos económicos y simbólicos dispares (Falabella, 2016) y están formadas por individuos y grupos que pueden tener diferentes intereses. En este sentido, desde los años 80 se ha producido un extenso cuerpo de literatura que subraya el papel de las escuelas como organizaciones micropolíticas (véase Bacharach \& Mundell, 1993; Ball, 1989; Blase, 1991; Hoyle, 1999; Marshall \& Scribner, 1991).

Con el concepto de micropolítica autores como Blase (1991) se refieren al ejercicio (sea formal o informal) del poder por parte de los individuos y de los grupos que pertenecen a la institución escolar, con el objetivo de conseguir sus propios fines en el seno de la escuela. Asimismo, Ball (1989) define la escuela como un campo de lucha formado por actores con distintos intereses e ideologías, que a veces se encuentran en conflicto. Así pues, podemos suponer que el proceso de implementación, lejos de resultar en una interpretación y aplicación directa y homogénea en todos los centros, puede derivar en una amplia variedad de aplicaciones y reinterpretaciones, tal y como sucedió con la recontextualización de la gestión de la diversidad en España (Bonal \& Rambla, 1999).

En la presente investigación se pretende analizar cómo se aplica la autonomía escolar y cómo ésta es "recontextualizada" (Bernstein, 1993) en diferentes contextos escolares. Para llevar a cabo esta tarea se parte de la noción de enactment que fue desarrollada por Ball, Maguire, \& Braun (2012) en la obra How schools do policy: policy enactment in secondary schools, donde estudian como las escuelas hacen las políticas en contextos sociales reales. El concepto de enactment se refiere al proceso de interpretación y traducción de las políticas en prácticas (es decir, a su recontextualización y aplicación) por parte de actores que operan en contextos reales. A través de esta obra se analiza el proceso dinámico de interpretación, apropiación y 
traducción de los textos políticos, huyendo de las concepciones simplistas del proceso de implementación y subrayando la importancia de concebir el proceso de enactment como un proceso complejo y dinámico en el que intervienen factores de diferente naturaleza. En otras palabras, en esta obra los autores entienden que el proceso de enactment no se produce en un vacío y que, en consecuencia, en éste pueden intervenir, por un lado, factores materiales, y, por otro lado, factores personales y sociales (Ball et al., 2012). Recientemente, distintos autores han utilizado el concepto de enactment para comprender mejor como las escuelas, y los actores que forman parte de ellas, hacen las políticas (Ball, Maguire, Braun, \& Hoskins, 2011; Braun, Ball, \& Maguire, 2011; Braun, Ball, Maguire, \& Hoskins, 2011; Hardy, 2013; Maguire, Hoskins, Ball, \& Braun, 2011; Thrupp \& Easter, 2012; Singh, Heimans, \& Glasswell, 2014).

Una de las principales omisiones de la literatura sobre la implementación de políticas educativas hace referencia al contexto de las escuelas. A menudo se omite que este puede jugar un rol mediador importante, ya sea facilitando o dificultando la aplicación de ciertas políticas. Cuando hablamos de contexto, autores como Ball et al. (2012) hacen referencia a cuestiones como la historia de los centros, los recursos e infraestructuras de que disponen, las presiones externas que reciben o las culturas profesionales. Autores como Thrupp y Lupton (2006) o Keddie (2014) señalan la relevancia del contexto en el que se sitúan las escuelas, para explicar las diferencias en los procesos internos, las interpretaciones y los resultados que se derivan de las políticas. Además, Thrupp y Lupton (2006) elaboran una crítica dirigida a aquellas teorías, como por ejemplo la NGP, que pretenden mejorar la eficiencia y la eficacia de las escuelas mediante la ingeniería organizacional, omitiendo la relevancia del contexto en el que estas se ubican. El presente estudio se centra principalmente en el contexto social y material de las escuelas.

\section{Metodología}

Las preguntas que orientan la investigación son: ¿Cómo se recontextualizan las ideas de la NGP en las escuelas públicas en Cataluña?; ¿Cómo se interpreta y aplica la autonomía escolar por parte de distintos actores?; ¿Cómo influye el contexto material y social del centro en el proceso 
de recontextualización de la autonomía escolar? En este sentido, la investigación pretende examinar el proceso de enactment de la autonomía escolar, es decir, su reinterpretación y aplicación por parte de distintos actores.

En segundo lugar, se explora la influencia del contexto social y material de la escuela, y, más concretamente, de la composición social del alumnado y la estabilidad de la plantilla docente, en la recontextualización de las ideas de autonomía escolar.

A nivel metodológico cabe destacar que la aproximación empírica al objeto de estudio consiste en el estudio de casos de tipo comparativo (Coller, 2005). Para ello se han seleccionado dos escuelas públicas de una ciudad media de Cataluña que operan en diferentes contextos materiales y sociales.

En relación a la elección del estudio de casos como método para la investigación, este se debe principalmente al potencial que nos ofrece (Coller, 2005), ya que, mediante la triangulación de las diferentes técnicas de investigación social y las fuentes, permite llegar a la convergencia de la evidencia (Yin, 2003).

En el marco de este estudio se analizan dos casos extremos. En cuanto a las características de las escuelas, si bien es cierto que ambas están desarrollando Planes de Autonomía de Centro (PAC), se diferencian por la composición social del alumnado (composición heterogénea y composición homogénea de clase trabajadora) y la estabilidad de la plantilla (plantilla estable y plantilla con elevada movilidad docente). En este sentido, la escuela Paulo Freire (en ambos casos se utilizan pseudónimos para garantizar el anonimato) es una escuela con elevada rotación del profesorado y una composición social homogénea de clase trabajadora y origen inmigrante. Por otro lado, la escuela Ferrer Guardia es una escuela con composición social heterogénea y una plantilla estable. De esta manera se quiere captar cómo inciden los factores contextuales en la implementación de la autonomía escolar. Por otra parte, las técnicas de investigación social que se utilizan consisten en entrevistas semi-estructuradas a la dirección escolar (2 entrevistas), el profesorado (4 entrevistas), a representantes de la Asociación de Madres y Padres (AMPA) de cada centro (2 entrevistas) y a la inspección (1 entrevista). Estas se complementan con un total de 34 cuestionarios que respondieron los docentes y el equipo directivo de cada centro. Por último, se 
ha aplicado la técnica de observación no participante en el claustro de cada escuela.

La información obtenida en la observación de los claustros fue anotada manualmente en un cuaderno de campo. Las anotaciones se hicieron siguiendo una estrategia narrativa que estuvo guiada por el uso de una plantilla de observación. Todas las entrevistas fueron grabadas y transcritas. El análisis de las entrevistas se realizó con el programa ATLAS.ti (versión 6) y combinó el uso de códigos preestablecidos y de códigos emergentes. En cuanto a los datos de las encuestas, estos fueron analizados con el programa SPSS (versión 18), mediante el cual se realizó un análisis descriptivo univariante.

\section{Marco analítico}

\section{Nueva gestión pública y autonomía escolar. Las tres dimensiones de la autonomía}

El concepto de autonomía escolar es un concepto multidimensional, hecho que facilita que se produzcan múltiples reinterpretaciones del mismo en el discurso político y, en consecuencia, que se utilice con diferentes fines. En la literatura se acostumbran a distinguir tres dimensiones de la autonomía escolar: pedagógica, organizativa y de gestión (véase Frías del Val, 2010; Larruzea, 2013). Sin embargo, estas se tienden a sintetizar en dos enfoques: el enfoque pedagógico y el enfoque gerencial, que incluye la dimensión organizativa y de gestión (Verger \& Curran, 2014).

La dimensión pedagógica de la autonomía incluye aquellas decisiones que hacen referencia al qué y el cómo se enseña. Concretamente, dentro de la autonomía pedagógica se incluyen esas decisiones vinculadas a los contenidos curriculares, la planificación, la metodología, la programación, los criterios de evaluación y el desarrollo de actividades docentes y complementarias, así como la formación y el desarrollo profesional de los docentes. Asimismo, mediante la autonomía pedagógica se pretende facilitar la adaptación metodológica y curricular a las características del entorno y del alumnado de cada centro (CEAE, 2009; Larruzea, 2013).

En cuanto al enfoque gerencial, este engloba cuestiones relacionadas con la dimensión organizativa, como por ejemplo: la organización de los 
espacios, el tiempo, los agrupamientos de los estudiantes, y la práctica docente de acuerdo con el proyecto de la escuela (CEAE, 2009). Por otro lado, también incluye aspectos relacionados con la gestión que se vinculan con el poder en la toma de decisiones en aspectos tales como la contratación de personal y servicios, la intervención en los procesos mediante los cuales se adscribe el profesorado al equipo docente de las escuelas, la creación de convenios y/o conciertos, y con la gestión económica, administrativa y material de los centros (Larruzea, 2013). Mediante la autonomía de gestión se quiere optimizar la eficiencia en la gestión de los recursos y contribuir a la mejora de los resultados (CEAE, 2009).

\section{El contexto escolar}

La presente investigación se ha desarrollado en dos escuelas de una ciudad media de la provincia de Barcelona. A continuación se describen las principales características de los distintos contextos escolares y seguidamente se presentan los principales resultados del estudio. En la Tabla 1 se pueden observar las principales características de ambas escuelas en relación con su contexto social y material.

\section{El contexto de la escuela Ferrer Guardia}

La escuela Ferrer Guardia ${ }^{3}$ es una escuela de primaria de dos líneas, que cuenta con unas instalaciones amplias, con un patio y aulas grandes, además de unos equipamientos tecnológicos que ofrecen muchas posibilidades.

En cuanto a la composición social del alumnado, hay que decir que tiene un porcentaje de alumnado de origen inmigrado bajo, que está alrededor del $4 \%$. Entre el alumnado de origen inmigrado hay un elevado número de estudiantes de nacionalidad italiana. El porcentaje de alumnos que ha solicitado becas comedor en el presente curso es del 5\%. En relación con el porcentaje total de alumnado con necesidades educativas especiales y específicas, también está alrededor del $5 \%$.

Por lo que se refiere al perfil social de las familias, los entrevistados consideran que se trata de una población que valora la escuela y que tiene una participación bastante activa en el ámbito escolar. 


\begin{tabular}{|c|c|c|}
\hline & Escuela Ferrer Guardia & Escuela Paulo Freire \\
\hline Composición del centro & $\begin{array}{l}\text { Composición social heterogénea: } \\
\text { alumnado mayoritariamente de } \\
\text { clase media y clase trabajadora, y } \\
\text { reducido porcentaje de alumnado } \\
\text { de origen inmigrante y con } \\
\text { Necesidades Educativas } \\
\text { Específicas o Especiales (NEE). }\end{array}$ & $\begin{array}{l}\text { Composición social homogénea: } \\
\text { alumnado de clase trabajadora y } \\
\text { origen inmigrante, con elevada } \\
\text { proporción de alumnado con } \\
\text { Necesidades Educativas Específicas } \\
\text { y Especiales. }\end{array}$ \\
\hline $\begin{array}{l}\text { Asociación de Madres y } \\
\text { Padres (AMPA) }\end{array}$ & $\begin{array}{l}\text { AMPA con una estructura fuerte y } \\
\text { burocratizada, que gestiona su } \\
\text { propio presupuesto y que organiza } \\
\text { una gran diversidad de actividades } \\
\text { en el centro. Los distintos actores } \\
\text { reconocen que se trata de una } \\
\text { AMPA muy activa y con capacidad } \\
\text { de incidir en el centro. El } 100 \% \text { de } \\
\text { la plantilla afirma que las familias } \\
\text { participan activamente en la } \\
\text { escuela. }\end{array}$ & $\begin{array}{l}\text { AMPA con una estructura débil que no } \\
\text { dispone de autonomía para gestionar } \\
\text { su propio presupuesto. } \\
\text { Tradicionalmente ha sido poco activa. } \\
\text { Su actividad se ha centrado en co- } \\
\text { organizar, juntamente con el equipo } \\
\text { docente, actividades de carácter } \\
\text { asistencial dirigidas a las familias y } \\
\text { alumnado de la escuela. El } 75 \% \text { de la } \\
\text { plantilla se muestra en desacuerdo en } \\
\text { que las familias participan } \\
\text { activamente en el centro. }\end{array}$ \\
\hline Instalaciones & $\begin{array}{l}\text { El centro se encuentra en medio de } \\
\text { un barrio residencial y cuenta con } \\
\text { unas infraestructuras amplias que } \\
\text { ofrecen un amplio abanico de } \\
\text { posibilidades, con aulas que } \\
\text { disponen de equipamiento } \\
\text { tecnológico moderno. }\end{array}$ & $\begin{array}{l}\text { El centro se sitúa en un barrio } \\
\text { separado del principal núcleo urbano } \\
\text { de la ciudad. El edificio de la escuela } \\
\text { está ubicado en el centro de un área } \\
\text { industrial y la fachada del edificio } \\
\text { parece antigua y deteriorada. } \\
\text { Recientemente empezaron a } \\
\text { incorporar nuevos equipamientos } \\
\text { tecnológicos en el centro. }\end{array}$ \\
\hline $\begin{array}{l}\text { Prestigio social del } \\
\text { centro educativo }\end{array}$ & $\begin{array}{l}\text { Escuela con elevado prestigio social } \\
\text { en el mercado educativo local: } \\
\text { escuela que tradicionalmente es } \\
\text { demandada en primera opción por } \\
\text { parte de las familias. }\end{array}$ & $\begin{array}{l}\text { Escuela con estigma social que tiene } \\
\text { escaso prestigió en el mercado } \\
\text { educativo local: escuela poco } \\
\text { demanda en primera opción debido al } \\
\text { fenómeno del "white flight". }\end{array}$ \\
\hline Rotación del profesorado & $\begin{array}{l}\text { Plantilla estable y con una larga } \\
\text { experiencia. }\end{array}$ & $\begin{array}{l}\text { Plantilla con elevada movilidad y con } \\
\text { docente que dificulta la consolidación } \\
\text { del proyecto educativo. }\end{array}$ \\
\hline
\end{tabular}

Tabla 1 - Características del contexto escolar

A nivel socioeconómico, los entrevistados afirman que las familias responden a un perfil social de clase media, ocupado en el sector servicios y en el tecnológico. Asimismo, la escuela disfruta de prestigio social en el espacio educativo local, de manera que acostumbra a ser demandada en primera opción por las familias, encontrándose a menudo con la situación de que hay una demanda superior al número de plazas ofrecidas. Es importante subrayar este aspecto, puesto que alrededor de la escuela hay poca población escolar, y la mayoría de familias que matriculan a sus hijos en la escuela en realidad tienen una escuela más cercana que ésta. 
A nivel organizativo, las familias del centro cuentan con una AMPA activa, que disfruta de una estructura consolidada con una elevada capacidad en la prestación de servicios, que se ve reforzada, por un lado, por una persona contratada para atender el servicio de comedor, y, por el otro, por una persona que gestiona los aspectos de tipo administrativo, como por ejemplo la economía de las actividades extraescolares, las cuotas de material, el comedor escolar y las salidas que se cobran a las familias, etc. La AMPA de la escuela habitualmente se reúne de manera quincenal y las convocatorias de las reuniones son públicas. El número de asistentes suele ser de 10012 personas de manera fija, si bien es cierto que el número de participantes acostumbra a ser mayor cuando se tienen que organizar acontecimientos puntuales.

Finalmente, en cuanto a la estabilidad de la plantilla de la escuela, está formada por docentes con una larga experiencia en el centro y que ocupan su plaza definitiva. Por lo tanto, la plantilla en términos generales es muy estable, ya que la mayoría de los maestros, excepto dos que están en comisión de servicios y uno que es interino, tienen plaza definitiva. En cuanto a la antigüedad media en el centro, esta es de unos 15 años aproximadamente.

\section{El contexto de la escuela Paulo Freire}

La escuela Paulo Freire dispone de unas instalaciones antiguas, formadas por cuatro edificios con diversas funcionalidades. Antes del curso 98-99 era de dos líneas, y aunque existía cierta diversidad, hay que señalar que la mayoría de la población matriculada en el centro era población autóctona procedente del mismo barrio, junto con una minoría de alumnado de etnia gitana. Con el paso de los años la composición social del alumnado del centro experimentó una profunda transformación, causada fundamentalmente por dos factores. Por un lado, la llegada de alumnado de origen inmigrante. Y, de la otra, "el efecto huida" o "white flight" (Nusche, 2009; Bonal, 2012) de las familias autóctonas, que se van hacia otras escuelas con una concentración menor de alumnado de origen inmigrante. En este sentido, según Waslander, Pater, y van der Weide (2010), las familias utilizan la composición social del centro como un indicador de calidad. La combinación de ambos elementos contribuyó a la eliminación de una línea, debida a la reducción de la matriculación de alumnado, que no respondía estrictamente a cuestiones sociodemográficas. 
En cuanto a la composición social del alumnado del centro, actualmente hay alrededor de un $75 \%$ de población de origen inmigrante. Los dos bloques mayoritarios están formados por alumnado de origen magrebí (28\%) y subsahariano $(27 \%)$ y el resto de alumnado de origen inmigrante es principalmente de origen paquistaní o latinoamericano. El porcentaje de alumnado autóctono es del $25 \%$, y mayoritariamente está formado por alumnado de etnia gitana. El porcentaje de alumnado con necesidades educativas específicas y especiales es del $80 \%$. En los últimos años, el alumnado con necesidades educativas específicas (por razones socioeconómicas) ha aumentado sustancialmente debido al efecto de la crisis económica. Ligado con esto, las becas comedor demandadas en el centro han pasado de 8 alumnos (curso 2012-2013) a más de 40 alumnos (curso 2013-2014).

En relación con el perfil social de las familias, los entrevistados consideran que mayoritariamente tienen un nivel socioeconómico muy bajo y/o precario. A su vez, están muy afectadas por el paro, ya que mayoritariamente trabajaban en el sector de la construcción, que ha sufrido una severa destrucción de ocupación a raíz de la crisis. A nivel cultural, se destaca que la mayoría de familias tienen bajos niveles de formación reglada, si bien es cierto que hay un reducido número de familias con educación secundaria obligatoria. En cuanto a la AMPA, esta se creó recientemente mediante la organización de algunas madres. De hecho, hay que decir que, desde la llegada del actual equipo directivo, se ha priorizado el acercamiento de las familias a la escuela. Desde la AMPA se organizan las fiestas de la escuela y talleres específicos (de catalán, de cocina, de violencia de género, etc.). Sin embargo, según los entrevistados ésta no es una AMPA con capacidad para gestionar los aspectos económicos.

Finalmente, en relación con la estabilidad de la plantilla, actualmente en el centro sólo hay 7 personas con plaza definitiva, en un centro que tiene alrededor de 20 docentes en plantilla. El resto de docentes están en comisiones de servicios, o bien ocupando lugares estructurales, lugares específicos y con reclamaciones. Por otro lado, en la escuela se habla de los "maestros fantasma", es decir, docentes que tienen plaza definitiva en el centro y que no los conocen ni los han visto nunca. Por lo que se refiere a la antigüedad media de los docentes del centro, es de 5 años. 


\section{Resultados: la recontextualización de la autonomía escolar en diferentes contextos}

\section{Jerarquización, burocratización y resistencias}

De acuerdo con la tesis de Ball et al. (2012), el proceso de aplicación de una política es un proceso creativo, dentro del cual se desarrollan distintas reinterpretaciones, prácticas y resistencias. Mediante las entrevistas con el profesorado de ambos centros se han observado múltiples resistencias docentes con causas diversas. Por un lado, se observaron resistencias frente a la introducción de la "cultura de la performatividad" (Ball, 2003). En los discursos de los docentes las medidas de rendición de cuentas asociadas al Plan de Autonomía de Centro (PAC) se percibían como un mecanismo de control sobre el trabajo docente, que generaba un aumento de la presión para conseguir los resultados (ENM01PF; ENM02FG; ENM05FG).

(...) te sientes presionado por qué tienes que pasar unas valoraciones, unas encuestas y unos no sé qué, y eso cómo lo valoraremos, y éste ítem cómo lo haremos, y todo eso y tal y cual. (ENM05FG)

Por otro lado, uno de los objetivos del PAC era superar el viejo modelo de gestión burocrática, mediante un nuevo modelo de gestión pública orientado, en parte, por la NGP (Garcia \& del Campo, 2012). Paradójicamente, se percibe que hay un aumento de la burocratización, como consecuencia de la implementación del PAC (ENM02FG; ENM05FG; ENF07FG; ENM01PF; END03PF).

La parte negativa del Plan de Autonomía es que como todo son papeles. Y... como todo, a veces te pide muchas cosas que tal vez... no hace falta tanta cosa y tanto papel. (ENM01PF)

Asimismo, en la entrevista con la dirección de la escuela Ferrer Guardia también se ha detectado una fuerte crítica al uso del discurso de la autonomía (END04FG), ya que considera que a menudo se utiliza como una "retórica vacía" (Larruzea, 2013). En este sentido, cabe recordar que el Plan de Autonomía de Centro (PAC), tal y como afirman las direcciones de ambos centros en las entrevistas, más que desarrollar la autonomía pedagógica, ha contribuido a introducir la Planificación Estratégica en el funcionamiento cotidiano de los centros, elemento que ha ido acompañado de otros recursos, como por ejemplo la red de centros PAC mediante la cual se comparten experiencias de éxito, el acompañamiento de la Inspección en el proceso de implementación, recursos económicos (sólo en el caso de los primeros 
centros PAC) y la prioridad a la hora de entrar en programas educativos promovidos por el Departamento de Educación de Cataluña.

Autores como Merchán (2012) advierten que, a pesar de que los discursos a menudo estén compuestos por una mezcla de conceptos como autonomía, liderazgo pedagógico, dirección, etc., mediante la introducción de la NGP se está produciendo una transformación del rol del director, que ve como se fortalece su posición en la toma de decisiones dentro del centro. En la presente investigación se ha observado el poder creciente de la dirección escolar, tanto en el ámbito de gestión como en la elaboración de documentos pedagógicos clave a nivel de centro, produciéndose un proceso de recentralización del poder dentro de la escuela. La triangulación de la evidencia obtenida a través de las distintas técnicas permite constatar la creciente relevancia de la dirección en el desarrollo de los documentos centrales de ambas escuela (PEC, Proyecto de Dirección, etc.). Asimismo, en ambos casos las direcciones afirman que tanto la figura del director como los equipos directivos han visto cómo sus responsabilidades aumentaban sustancialmente con el Decreto de Direcciones (END04FG; END03PF).

(...) mis responsabilidades han aumentado no únicamente con el PAC, con el Decreto de Dirección todas las responsabilidades recaen cada vez más sobre el director. (END03PF)

En cuanto a la elaboración del Proyecto Educativo de Centro (PEC), en los discursos de los maestros de ambos centros se señala que tanto el PEC como el Proyecto de Dirección son percibidos como documentos burocráticos ajenos a la práctica docente que se desarrolla a nivel de aula, mostrando un desconocimiento del contenido de los mismos (ENM05FG; ENM06PF; ENM01PF).

Mira, el PEC, sinceramente, se hizo más desde la dirección. O sea, ya partíamos del PEC que había antes en la escuela. Se habló en un claustro. Sí que se hicieron algunas aportaciones, pero al final el documento final lo hizo la dirección... las características del PEC no sabría cómo explicarlas... (ENM01PF)

En este sentido, Larruzea (2013) señala que una de las principales dificultades para la aplicación y el desarrollo de la autonomía pedagógica reside en el hecho de que el PEC de las escuelas a menudo es débil o prácticamente no existe, lo que genera que en muchos casos se convierta en una simple formalidad de carácter burocrático, que generalmente es olvidada por el personal del centro. 


\section{Reinterpretación y apropiación de la autonomía en diferentes contextos escolares}

A pesar de observarse algunas resistencias y tendencias comunes en la recepción de la autonomía por parte de los docentes y los equipos directivos de ambos centros, como veremos, el contexto escolar juega un rol importante en la adopción de respuestas diferenciales relacionadas con la reinterpretación, la apropiación y la aplicación de la autonomía escolar. A continuación se presenta una tabla (Tabla 2) que tiene por objetivo mostrar las respuestas diferenciales que se ofrecen frente a la autonomía escolar en función del contexto social y material de las escuelas donde se aplica.

\begin{tabular}{|c|c|c|}
\hline & Escuela Ferrer Guardia & Escuela Paulo Freire \\
\hline \multirow[t]{2}{*}{$\begin{array}{l}\text { Reinterpretación y } \\
\text { apropiación de la } \\
\text { autonomía escolar }\end{array}$} & $\begin{array}{l}\text { Diferentes significados para el } \\
\text { director (que pone énfasis en el } \\
\text { enfoque gerencial de la autonomía) } \\
\text { y los docentes (que enfatizan la } \\
\text { dimensión pedagógica). }\end{array}$ & $\begin{array}{l}\text { Adhesión, por parte del director y los } \\
\text { docentes, a la dimensión gerencial de } \\
\text { la autonomía escolar. En el caso de } \\
\text { los docentes, consideran que los } \\
\text { aspectos gerenciales de la autonomía } \\
\text { son los más importantes debido al } \\
\text { contexto escolar y a la elevada } \\
\text { inestabilidad de la plantilla que } \\
\text { genera. }\end{array}$ \\
\hline & $\begin{array}{l}\text { La estabilidad de la plantilla facilita } \\
\text { la consolidación del proyecto } \\
\text { educativo de centro. }\end{array}$ & $\begin{array}{l}\text { Dificultades para consolidar el } \\
\text { proyecto educativo de centro debido } \\
\text { al contexto escolar. }\end{array}$ \\
\hline $\begin{array}{l}\text { Aplicación de la } \\
\text { autonomía pedagógica }\end{array}$ & $\begin{array}{l}\text { Los docentes consideran que el } \\
\text { perfil social del alumnado facilita la } \\
\text { aplicación y el desarrollo de la } \\
\text { autonomía pedagógica. }\end{array}$ & $\begin{array}{l}\text { Los docentes señalan que el contexto } \\
\text { social del centro dificulta el desarrollo } \\
\text { de la autonomía pedagógica. Se } \\
\text { observan discursos sobre el "déficit } \\
\text { familiar" y una reducción de } \\
\text { expectativas docentes y de los } \\
\text { estándares de aprendizaje. }\end{array}$ \\
\hline Trabajo en el aula & $\begin{array}{l}\text { Pueden dedicarse a tareas de } \\
\text { enseñanza y aprendizaje. }\end{array}$ & $\begin{array}{l}\text { Una parte importante del tiempo lo } \\
\text { invierten a la resolución de conflictos } \\
\text { en el aula. }\end{array}$ \\
\hline $\begin{array}{l}\text { Tiempo para preparar } \\
\text { las clases }\end{array}$ & $\begin{array}{l}66,7 \% \text { de los docentes muestra } \\
\text { satisfacción con el tiempo de que } \\
\text { dispone para preparar las clases. }\end{array}$ & $\begin{array}{l}75,1 \% \text { de los docentes considera que } \\
\text { el tiempo de que dispone para } \\
\text { preparar las clases es insuficiente. }\end{array}$ \\
\hline $\begin{array}{l}\text { Percepción de impacto } \\
\text { positivo sobre el } \\
\text { alumnado }\end{array}$ & $\begin{array}{l}\text { El } 100 \% \text { de los docentes se } \\
\text { muestra de acuerdo o muy de } \\
\text { acuerdo en que tienen un impacto } \\
\text { positivo sobre sus alumnos }\end{array}$ & $\begin{array}{l}\text { El } 93,7 \% \text { de los docentes se muestra } \\
\text { de acuerdo o muy de acuerdo en que } \\
\text { tienen un impacto positivo sobre sus } \\
\text { alumnos. Sin embargo, el } 6,3 \% \text { se } \\
\text { muestra en desacuerdo. }\end{array}$ \\
\hline $\begin{array}{l}\text { Satisfacción con los } \\
\text { materiales y recursos } \\
\text { educativos del centro }\end{array}$ & $\begin{array}{l}\text { Los docentes muestran una elevada } \\
\text { satisfacción con los materiales y } \\
\text { recursos educativos de los que } \\
\text { disponen }(93,3 \%) \text {. }\end{array}$ & $\begin{array}{l}\text { El } 37,5 \% \text { de los docentes considera } \\
\text { que los materiales y recursos } \\
\text { educativos de que disponen no son } \\
\text { adecuados. }\end{array}$ \\
\hline $\begin{array}{l}\text { Los docentes han } \\
\text { pensado buscar trabajo } \\
\text { en otras escuelas }\end{array}$ & $\begin{array}{l}\text { El } 100 \% \text { de los docentes se } \\
\text { muestran en desacuerdo. }\end{array}$ & $\begin{array}{l}\text { El } 28 \% \text { se muestra de acuerdo y } \\
\text { afirma haber pensado en buscar } \\
\text { trabajo en otros centros. }\end{array}$ \\
\hline
\end{tabular}


Entrando en la reinterpretación y apropiación de la autonomía pedagógica por parte de los centros, en el caso de la escuela Ferrer Guardia se observa que los docentes enfatizan la dimensión pedagógica de la autonomía (ENM05FG; ENM02FG).

Para mi [la autonomía escolar] es el hecho de que, a partir de unas directrices marco, la escuela pueda ajustar tanto los contenidos, las metodologías (...) y poder gestionarlos realmente, autogestionar en función de la población que tienes. (ENM02FG)

En cambio desde la dirección, se subraya que el PAC ha favorecido el desarrollo de aspectos gerenciales, mediante la definición de los perfiles de los docentes en los procesos de selección, y a su vez ha facilitado la obtención de recursos económicos que han permitido remodelar los espacios físicos de la escuela (END04FG). Sin embargo, tanto los docentes como la dirección hacen referencia al hecho de que recogen y comparten todo lo que hacen en el aula. Así mismo, destacan que los aspectos curriculares generalmente se trabajan a nivel de ciclo y en reuniones interciclo a través de las cuales es posible articular una visión global a nivel de escuela para analizar qué cuestiones se pueden mejorar (ENM02FG; END04FG; ENM05FG). Por otro lado, es importante subrayar que hay una armonización de los criterios de evaluación a través de las llamadas "rúbricas", que en palabras de la dirección significan:

[Ir] un paso más allá de lo que sería el proceso de la evaluación, para garantizar elementos de coincidencia en los criterios que usamos para evaluar y que todos [los docentes del centro] tengan una información clara sobre qué criterios evaluamos, teniendo en cuenta que son unas rúbricas que tenemos en cuenta todos los maestros, con un mismo criterio (...) entonces es toda una estructura para garantizar que todo el personal actué con los mismos criterios, pero tienen que estar constituidas, colgadas en un lugar, e incrustadas también en la manera de actuar. Se trata de ir poco a poco pero a paso firme. (END04FG)

Otra muestra de la apropiación de la autonomía a nivel pedagógico es el hecho que desde el equipo docente se considera que la autonomía ha aumentado la reflexividad sobre la práctica que los maestros llevan a cabo, la cual permite una reflexión crítica alrededor de la praxis docente.

Es una renovación mucho más constante. Tienes que tener muy claro que las actividades del Proyecto de Autonomía son 3 o 4, que tienes que rendir cuentas, por lo tanto, tienes que procurar que haya unos resultados, valorar sino los consigues porque no los has conseguido, o porque han funcionado, porque no 
han funcionado. Es decir, te trae mucha más reflexión a nivel docente de tu trabajo en el aula... el Plan de Autonomía del alumnado con proyectos... ¿Funcionan los proyectos? ¿Por qué nos han funcionado? ¿Por qué no? ¿Qué ha fallado? Conlleva más trabajo de coordinación con los compañeros... más debate... pero personalmente creo que es interesante la discusión de las maneras de trabajar. (ENM02FG)

A diferencia de la escuela Ferrer Guardia, en la escuela Paulo Freire hay una mayor adhesión hacia los aspectos gerenciales, incluso por parte de los docentes (ENM01PF; END03PF). Esto en parte es consecuencia del contexto de la escuela, marcado por una elevada segregación escolar y por la existencia de una plantilla docente inestable.

La autonomía escolar para mí... es el hecho de, como equipo directivo y como escuela, poder decidir ciertas cosas. No depender tanto de... por ejemplo, con eso que te decía de las plantillas, ahora hay mucha polémica por el decreto de plantillas, que yo lo entiendo, pero en nuestro caso, yo creo que es muy importante que el equipo directivo pueda... si hay maestros que han venido aquí a hacer una sustitución o como interinos y han funcionado, que se puedan quedar... Autonomía también... no lo sé... para mí, y te lo digo desde el punto de vista del lugar donde estamos, por ejemplo, lo más importante es esto, a la hora de decidir la plantilla de la escuela. (ENM01PF)

A pesar de considerar que la dimensión gerencial de la autonomía es la más importante, los docentes señalan que en la escuela también se trabajan las cuestiones pedagógicas en diferentes niveles (ciclos, interciclos y claustros), siguiendo siempre una línea pedagógica y una manera de actuar, a lo que hay que añadir la coordinación en la unificación de criterios metodológicos (ENM01PF; END06PF). Sin embargo, a diferencia de la escuela Ferrer Guardia, en la escuela Paulo Freire la plantilla docente reconoce que el contexto escolar, y la consiguiente inestabilidad de la plantilla que conlleva, dificulta la consolidación del proyecto pedagógico y el trabajo colegiado y colaborativo entre diferentes niveles y entre docentes. En este sentido, en el claustro se observó que esta cuestión generaba ansiedad entre los docentes, mientras que el equipo directivo expresaba las dificultades para consolidar un proyecto educativo en este escenario de incertidumbre e inestabilidad (OBSCL2).

En relación con la influencia del contexto en el proceso de aplicación de la autonomía pedagógica, en la escuela Ferrer Guardia se señala que el perfil de los alumnos es muy "agradecido" y los maestros están muy a gusto 
porque pueden dedicar mucho tiempo en tareas vinculadas al aprendizaje del alumnado.

Realmente, aquí han venido maestros de otros lugares y verdaderamente lo que dicen es que esta es una escuela en la que se agradece trabajar. Que están a gusto (...) y que se puede dedicar tiempo al aprendizaje. (END04FG)

En cambio, en el caso de la escuela Paulo Freire, el contexto socioeducativo es totalmente distinto al de la escuela Ferrer Guardia. En este caso, existe una realidad social más compleja que genera que los docentes tengan que dedicar más tiempo a la resolución de conflictos en el aula, y, por ende, puedan dedicar menor tiempo al aprendizaje del alumnado:

Nosotros algún año nos hemos encontrado con algún maestro que decía: jufff! ¡Que cansinos que son estos niños! O bien... ¡ icon estos niños no se puede hacer nada! (END03PF)

Del mismo modo, en la escuela Paulo Freire hay un porcentaje de docentes muy superior (75\%) que afirma estar en desacuerdo en que disponen de tiempo suficiente para preparar las clases, mientras que en el caso de la escuela Ferrer Guardia sólo hay un 33,7\% de los docentes que se muestran en desacuerdo. El efecto contexto también se ve con la satisfacción con los materiales y recursos educativos de los que disponen los docentes. En la escuela Ferrer Guardia un $93,4 \%$ de los docentes se muestran satisfechos con los materiales y recursos educativos, mientras que en la escuela Paulo Freire el porcentaje de docentes que muestran insatisfacción en este aspecto asciende a cerca del $40 \%$.

Asimismo, en las entrevistas con la dirección y los docentes de la escuela Paulo Freire, vemos cómo se justifica la dificultad de desarrollar la autonomía pedagógica (y el trabajo por proyectos en particular) en base a la baja implicación familiar en la educación, como consecuencia de la situación de precariedad que afecta a las familias, adhiriéndose así a las "tesis de la cultura de la pobreza" (Lewis, 1987, cit. por Bonal \& Tarabini, 2010). La presencia de esta tesis en el discurso del profesorado se hace patente al hablar de la autonomía pedagógica y la dificultad de llevarla a cabo con éxito debido al contexto socioeconómico en el que vive el alumnado:

A ver, antes que nada, claro, no es un tipo de escuela... dijéramos... de las normalizadas, ya empezando por aquí. Entonces, es una escuela con unas dificultades en un entorno muy concreto. $Y$ donde las familias tienen unas 
dificultades económicas y hay mucha inmigración. Nosotros, pedagógicamente, en la línea de escuela intentamos trabajar a partir de proyectos con una metodología bastante participativa, pero nos es bastante difícil por el tipo de alumnado, porque las familias no se implican y entonces... cuando pedimos un poco de trabajo fuera de la escuela o que traigan cosas, que busquen cosas... cuesta mucho. (ENM01PF)

En relación con el punto anterior se observan coincidencias entre la evidencia cualitativa y la cuantitativa. De este modo, a nivel cuantitativo también se constata claramente la diferencia en la percepción que tienen los docentes alrededor de la implicación familiar. Así pues, mientras que en la escuela Ferrer Guardia la mayoría de los maestros se muestra de acuerdo $(86,67 \%)$ o muy de acuerdo $(13,33 \%)$ en que las familias de la escuela participan activamente en la dinámica de la escuela, en el caso de la escuela Paulo Freire el $75 \%$ de los maestros se muestra en desacuerdo $(31,25 \%)$ o muy en desacuerdo $(43,75 \%)$ con esta afirmación.

A estos factores hay que añadir el hecho de que la movilidad docente es superior en la escuela Paulo Freire. En este punto, en el análisis cuantitativo se ha observado que, a diferencia de la escuela Ferrer Guardia, donde el $100 \%$ de los docentes afirman estar en desacuerdo en que han pensado buscar trabajo en otras escuelas, en la escuela Paulo Freire hay un porcentaje importante de docentes (28\%) que se ha planteado buscar trabajo en otros centros. En este sentido, tal y como se ha señalado, la elevada movilidad docente crea un clima de incertidumbre en el centro y genera mayores dificultades para construir una plantilla docente cohesionada, desarrollar la autonomía pedagógica y consolidar el proyecto educativo del centro (OBSCL2).

Por otro lado, hay que señalar que la existencia de un contexto tan desfavorecido y segregado genera el peligro de una cosificación de las desigualdades educativas, que se traduce en unas expectativas y objetivos educativos menores en el caso de la escuela Paulo Freire.

Mira, el currículum está, obviamente. Pero aquí lo tenemos todo muy adaptado. Quiero decir, el nivel que tenemos en esta escuela no es el que tenemos en otra escuela. Entonces, hicimos esto, decidimos, por ejemplo, de matemáticas y de lengua, poner unos mínimos a cada ciclo, o para cada curso. Entonces, son cosas básicas que se tienen que trabajar y esto lo hacemos acá que tenemos el Plan de Autonomía. Porque ya te digo, el nivel es más bajo y entonces hemos tenido que bajar los objetivos. (ENM06PF) 
De hecho, en este caso parte de las resistencias docentes frente a la introducción de la cultura de la performatividad a través de la rendición de cuentas y la autoevaluación son debidas a que consideran que el alumnado de su escuela nunca podrá alcanzar los resultados académicos de la media de Cataluña, y que, por lo tanto, es injusto que los evalúen en base a los resultados y los comparen con otros centros. Autores como Merchán (2012) han señalado que a menudo medidas como la autoevaluación (en el marco de la NGP) tienden a delegar las responsabilidades de posibles fracasos en las escuelas, que son tratadas como si fueran instituciones totalmente descontextualizadas (Wrigley, 2007, cit. por Merchán, 2012), ignorando pues, "el carácter sistémico de los problemas educativos y la incidencia de factores sobre los que la escuela no tiene capacidad de intervención" (Merchán, 2012, p. 15). En este sentido, una docente señala que:

[en el Plan de Autonomía]... así como la evaluación por un lado podría ser la parte positiva, por otro lado, a veces te da la sensación que te están evaluando por los resultados educativos de la escuela, y los resultados educativos en este caso no lo son todo. Y que obviamente nosotros nunca estaremos ni de lejos en la media de Cataluña, ni de España, ni de Europa. Y a veces siempre te comparan con la media y esto te deprime un poco, porque nosotros no llegaremos. (ENM01PF)

A modo de síntesis, en el presente artículo se han identificado algunas tendencias y respuestas diferenciales frente a la autonomía escolar, condicionadas por el contexto social del centro en el que se aplica. En cuanto a la reinterpretación de la autonomía escolar, si bien es común que los directores enfaticen más la dimensión gerencial, en el caso de los docentes se observan diferencias, de manera que, mientras que en la escuela Ferrer Guardia se apropian de la dimensión pedagógica de la autonomía, en el caso de la escuela Paulo Freire hay una mayor apropiación de la dimensión gerencial, hecho que se vincula directamente con el contexto social del centro y a la elevada rotación del profesorado. Estas mismas circunstancias dificultan la consolidación de un proyecto educativo de centro, mientras que en el caso de la escuela Ferrer Guardia sucede todo lo contrario.

En cuanto a la aplicación de la autonomía pedagógica, en la escuela Ferrer Guardia sostienen que el perfil social del alumnado facilita la aplicación y el desarrollo de la autonomía pedagógica, así como el trabajo en el aula y la dedicación a tareas de enseñanza. En cambio, en la Escuela Paulo Freire los 
docentes perciben que el contexto dificulta el desarrollo de la autonomía pedagógica y se observa una reducción de las expectativas docentes y de los estándares de aprendizaje exigidos al alumnado. Además, consideran que una parte importante del tiempo de clase deben dedicarlo a cuestiones disciplinarias, en detrimento del tiempo dedicado a la enseñanza.

Por último, el contexto escolar también incide sobre la satisfacción docente en relación con el tiempo para preparar las clases, los materiales y recursos educativos de que dispone el centro y las expectativas de continuar trabajando en el centro (véase Tabla 2).

\section{Conclusiones}

La NGP, y particularmente la autonomía escolar, ha tenido una gran influencia en las reformas educativas que se han iniciado en la última década en los países del sur de Europa. Tanto en el estado español, como en Cataluña, estas reformas han suscitado un enorme debate social y político. No obstante, a pesar de la evidencia internacional, se necesita más investigación sobre temas como por ejemplo: ¿Cómo funcionan las políticas de autonomía escolar en diferentes contextos? ¿Qué impacto tiene la autonomía escolar en la equidad del sistema educativo? ¿Qué impacto tiene la implementación de la autonomía escolar en la gobernanza de los centros educativos? ¿Qué efectos tiene la autonomía escolar sobre el desarrollo profesional y la satisfacción docente?

En el presente estudio exploratorio se ha hecho una aproximación empírica a la recontextualización de la autonomía escolar en la escuela pública en Cataluña, con el fin de dilucidar de qué manera el contexto de la escuela influye en la reinterpretación, apropiación y aplicación que los distintos actores hacen de esta política. Por otro lado, también se han señalado los posibles efectos en relación con la distribución de poder (entre docentes y directivos) en las escuelas, donde se observa un aumento de la relevancia de la figura del director.

Además, también se han señalado posibles efectos paradójicos (por ejemplo, el aumento de la burocratización) y perversos. Escudándose en la evidencia que procede de PISA, muchos gobiernos están promoviendo reformas de la NGP, que incluyen la autonomía escolar. Es cierto que PISA 
muestra una asociación entre la autonomía pedagógica de los centros y el rendimiento académico del alumnado. Sin embargo, el presente análisis indica que la autonomía escolar, si no se acompaña de políticas educativas de carácter estructural que combinen adecuadamente la lucha contra la desigualdad y la segregación escolar, con políticas focalizadas que brinden un apoyo especial a los centros más vulnerables, puede tener riesgos para la calidad de los centros y para la equidad educativa. Es decir, si la autonomía escolar no se acompaña de políticas sistémicas orientadas hacia la equidad existe el riesgo de cosificar las desigualdades educativas y aumentar la brecha en la calidad de los centros que forman parte de la red pública (Larruzea, 2013).

\section{Notas}

1 El autor desea expresar su agradecimiento por los comentarios y aportaciones a Antoni Verger, Geo Saura, Mauro Moschetti y a los dos revisores anónimos.

2 El código ENINSDEPEDUCO9 se refiere a la entrevista realizada con el inspector de las escuelas.

3 En ambos casos se utilizan pseudónimos para garantizar el anonimato de las escuelas.

\section{Referências}

Bacharach, S., \& Mundell B. (1993). Organizational politics in school: Micro, macro, and logics of action. Educational Administration Quarterly, 29(9), 423-452.

Ball, S. J. (1989). La micropolítica de la escuela. Hacia una teoría de la organización escolar. Madrid: Paidós/MEC.

Ball, S. J. (2003). Profesionalismo, gerencialismo y performatividad. Educación y Pedagogía, 37(15), 87-104.

Ball, S. J., \& Youdell, D. (2007). Hidden privatisation in public education. Brussels: Education International.

Ball, S. J., Maguire, M., \& Braun, A. (2012). How schools do policy: Policy enactment in secondary schools. New York: Routledge.

Ball, S. J., Maguire, M., Braun, A., \& Hoskins, K. (2011). Policy actors: Doing policy work in schools. Discourse: Studies in the Cultural Politics of Education, 32(4), 625639.

Barroso, J. (2004). A autonomia das escolas: Uma ficção necessária. Revista Portuguesa de Educação, 17(2), 49-83. 
Barroso, J. (2005). Liderazgo y autonomía de los centros educativos: Liderazgo y educación. Revista Española de Pedagogía, 63(232), 423-441.

Barzelay, M. (2001). La nueva gerencia pública. Un ensayo bibliográfico para estudiosos latinoamericanos (y otros). Revista del CLAD Reforma y Democracia, 19, 1-35.

Bernal, J. L., \& Lorenzo J. (2012). La privatización de la educación pública. Una tendencia en España. Un camino encubierto hacia la desigualdad. Profesorado: Revista de Currículum y Formación del Profesorado, 16(3), 81-109.

Bernal, J. L., Cano, J., \& Lorenzo, J. (2014). Organización de los centros educativos. LOMCE y políticas neoliberales. Zaragoza: Mira Editores.

Bernstein, B. (1993). La estructura del discurso pedagógico. Madrid: Ediciones Morata.

Blase, J. (1991). The politics of life in schools: Power, conflict, and cooperation. Newbury Park: Sage.

Bolívar, A. (2001). Liderazgo educativo y reestructuración escolar. In Actas del I Congreso Nacional sobre Liderazgo en el Sistema Educativo Español (pp. 95130). Córdoba: Departamento de Educación de la Universidad de Córdoba.

Bonal, X. (2012). Education policy and school segregation of migrant students in Catalonia: The politics of non-decision-making. Journal of Education Policy, 27(3), 401-421.

Bonal, X., \& Rambla, X. (1999). The recontextualisation process of educational diversity: New forms to legitimise pedagogic practice. International Studies in Sociology of Education, 9(2), 195-214.

Bonal, X., \& Tarabini, A. (Dir.) (2010). Ser pobre en la escuela. Habitus de pobreza y condiciones de educabilidad. Buenos Aires: Miño y Dávila.

Bonal, X., \& Tarabini, A. (2013). The role of PISA in shaping hegemonic educational discourses, policies and practices: The case of Spain. Research in Comparative and International Education, 3, 335-341.

Bonal, X., \& Verger, A. (2013a). Les opcions de política educativa a Catalunya: Anàlisi de l'acció de govern (2010-2013). In M. Martínez \& B. Albaigés (Dir.), L'estat de l'educació a Catalunya. Anuari 2013. Barcelona: Fundació Jaume Bofill. Enlace: http://www.gepsuab.cat/sites/default/files/publicacionsadjunts/9_Les_ opcions_de_politica_educativa_WEB.pdf

Bonal, X., \& Verger, A. (2013b). L'agenda de la política educativa a Catalunya: Una anàlisi de les opcions de govern (2011-2013). Barcelona: Fundació Jaume Bofill. Enlace: http://www.fbofill.cat/intra/fbofill/documents/publicacions/587.pdf

Braun, A., Ball, S. J., \& Maguire, M. (2011). Policy enactments in schools introduction: Towards a toolbox for theory and research. Discourse: Studies in the Cultural Politics of Education, 32(4), 581-583.

Braun, A., Ball, S. J., Maguire, M., \& Hoskins, K. (2011). Taking context seriously: Towards explaining policy enactments in the secondary school. Discourse: Studies in the Cultural Politics of Education, 32(4), 585-596.

Calero, J. (2010). La autonomía de los centros escolares y su relación con la equidad. CEE Participación Educativa, 13, 88-94. 
CEAE. (2009). La autonomía de los centros educativos como factor de calidad. Documento 1. XX Encuentro de Consejos Escolares y del Estado. Enlace: http://www.consejoescolardecantabria.es/PDF/ 4.2.4.ENCUENTROS_1.pdf Coller, X. (2005). Estudio de casos. Madrid: CIS.

Common, R. K. (1998). Convergence and transfer: A review of the globalisation of new public management. International Journal of Public Sector Management, 11(6), 440-450.

Espínola, V. (2000). Autonomía escolar: Factores que contribuyen a una escuela más efectiva. Inter-American Development Bank. Enlace: http://www.iadb.org/ wmsfiles/products/publications/documents/1017850.pdf

Estruch, J. (2006). Autonomía ¿para qué? Cuadernos de Pedagogía, 362, 104-108.

Falabella, A. (2016). ¿Qué aseguran las políticas de aseguramiento de la calidad? Un estudio de casos en distintos contextos escolares. Revista Estudios Pedagógicos, 41(1), 107-126.

Frías del Val, A. (2010). La autonomía de los centros docentes: Un equilibrio. Participación Educativa, 13, 42-61.

García, M. (2005). Culturas de enseñanza y absentismo escolar en la enseñanza secundaria obligatoria: Estudio de casos en la ciudad de Barcelona. Revista de Educación, 338, 347-374.

Garcia, E., \& del Campo, M. (2012). ¿La corresponsabilidad es una estrategia de éxito?. Revista de Educación, número extraordinario, 220-248.

Grimaldi, E., \& Serpieri, R. (2013). Jigsawing education evaluation. Pieces from the Italian new public management puzzle. Journal of Educational Administration and History, 45(4), 306-335.

Gunter, H. M. (2001). Leaders and leadership in education. London: SAGE Publications.

Hardy, I. (2013). A logic of appropriation: Enacting national testing (NAPLAN) in Australia. Journal of Education Policy, 29(1), 1-18.

Hartley, D. (2001). El Estado evaluador y la autonomía de la educación: ¿Motivo de reflexión? In J. Smyth (Ed.), La autonomía escolar: Una perspectiva crítica (pp. 119-137). Madrid: Akal.

Hood, C. (1991). A public management for all seasons?. Public Administration, 69(1), 3-19.

Hoyle, E. (1999). The two faces of micropolitics, School Leadership \& Management, 19(2), 213-222.

Keddie, A. (2014). It's like Spiderman... with great power comes great responsibility: School autonomy, school context and the audit culture. School Leadership \& Management, 34(5), 502-517.

Landri, P. (2009). A temporary eclipse of bureaucracy. The circulation of school autonomy in Italy. Italian Journal of Sociology of Education, 3(3), 76-93.

Larruzea, G. (2013). La autonomía de los centros escolares. Análisis y propuestas en clave de servicio público y equidad. El Ejido: Editorial Círculo Rojo.

Luengo, J. J., \& Saura, G. (2012). Mecanismos endógenos de privatización encubierta en la escuela pública. Políticas educativas de gestión de resultados y rendición 
de cuentas en Andalucía. Profesorado: Revista de Currículum y Formación del Profesorado, 16(3), 111-122.

Maguire, M., Hoskins, K., Ball, S., \& Braun, A. (2011). Policy discourses in school texts. Discourse: Studies in the Cultural Politics of Education, 32(4), 597-609.

Maragall, E., \& Colomé, F. (2013). Escola Nova, poble lliure. Barcelona: RBA.

Marshall, C., \& Scribner, J. D. (1991). It's all political: Inquiry into the micropolitics of education. Education and Urban Society, 23(4), 347-355.

Merchán, F. J. (2012). La introducción en España de la política educativa basada en la gestión empresarial de la escuela: El caso de Andalucía. Archivos Analíticos de Políticas Educativas, 20(32), 1-28.

Meuret, D. (2004). La autonomía de los centros escolares y su regulación. Revista de Educación, 333, 11-19.

Neave, G. (1998). The evaluative state reconsidered. European Journal of Education, 3(3) 265-284.

Nusche, D. (2009). What works in migrant education? A review of evidence and policy options. OECD Education Working Papers, 22. OECD Publishing.

OCDE (2009). TALIS (OCDE). Estudio internacional sobre la enseñanza y el aprendizaje. Informe español 2009. Madrid: Ministerio de Educación.

OCDE (2011). PISA in focus 9: School autonomy and accountability: Are they related to student performance?. Paris: OECD. Enlace: http://www.oecd.org/pisa/ pisaproducts/pisainfocus/48910490.pdf

Pont, B., Nusche, D., \& Moorman, H. (2009). Mejorar el liderazgo escolar, Volumen 1: Política y práctica. OECD Publishing.

Sahlberg, P. (2006). Education reform for raising economic competitiveness. Journal of Educational Change, 7, 259-287.

Sancho, M., A. (2014). Posición de las comunidades autónomas ante la autonomía escolar de los centros públicos. Revista de Educación, 366, 64-86.

Saura, G. (2015). Think tanks y educación. Neoliberalismo de FAES en la LOMCE. Education Policy Analysis Archives, 23(107), 1-16.

Sehested, K. (2002). How new public management reforms challenge the roles of professionals. International Journal of Public Administration, 25(12), 1513-1537.

Singh, P., Heimans, S., \& Glasswell, K. (2014). Policy enactment, context and performativity: Ontological politics and researching Australian National Partnership policies. Journal of Education Policy, 29(6), 826-844.

Spillane, J. P., Halverson, R., \& Diamond, J. B. (2001). Investigating school leadership practice: A distributed perspective. Educational Researcher, 23-28.

Tarabini, A., Curran, M., Montes, A., \& Parcerisa, L. (2015). El rol de los centros educativos en la prevención del abandono escolar: Una aproximación desde la perspectiva micropolítica. Educação, Sociedade \& Culturas, Special Issue: Education Policies and Early School Leaving, 45, 121-141.

Thrupp, M., \& Easter, A. (2012). Research, analysis and insight into national standards (RAINS) project. Hamilton: University of Waikato. 
Thrupp, M., \& Lupton, R. (2006). Taking school contexts more seriously: The social justice challenge. British Journal of Educational Studies, 54(3), 308-328.

Tolofari, S. (2005). New Public Management and education. Policy Futures in Education, 3(1), 75-89.

Verger, A. (2007). L'AGCS i la política de l'educació: Anàlisi dels factors de liberalització (Tesis doctoral). Universitat Autónoma de Barcelona, Bellaterra. Enlace: http://ddd.uab.cat/pub/tesis/2007/ tdx-0229108-170149/avp1de1.pdf

Verger, A. (2012). Globalización, reformas educativas y la nueva gestión del personal docente. Revista Docencia, 46, 4-13.

Verger, A., \& Altineyelken, H. (2013). Global education reforms and the new management of teachers: A critical introduction. In A. Verger, H. Altineyelken, \& M. Koning (Eds.), Global managerial education reforms and teachers: Emerging policies, controversies and issues in developing contexts (pp. 1-18). Brussels: Education International Research Institute.

Verger, A., \& Curran, M. (2014). New Public Management as a global education policy: Its adoption and re-contextualization in a southern European setting. Critical Studies in Education, 55(3), 253-271.

Verger, A., Curran, M., \& Parcerisa, L. (2015). La trayectoria de una reforma educativa global: El caso de la Nueva Gestión Pública en el sistema educativo catalán. Educação \& Sociedade, 36(132), 675-697.

Verger, A., \& Normand, R. (2015). Nueva Gestión Pública y educación: Elementos teóricos y conceptuales para el estudio de un modelo de reforma educativa global. Educação \& Sociedade, 36(132), 599-622.

Waslander, S., Pater, C., \& van der Weide, M. (2010). Markets in education: An analytical review of empirical research on market mechanisms in education. OECD Education Working Papers, 52, OECD Publishing. doi: 10.1787/5km4pskmkr27-en.

Wittmann, E. (2008). Align, don't necessarily follow. Educational Management Administration \& Leadership, 36(1), 33-54.

Yin, R. (2003). Case study research: Design and methods. California: SAGE Publications. 


\title{
NEW PUBLIC MANAGEMENT AND EDUCATION REFORM: THE RECONTEXTUALIZATION OF SCHOOL AUTONOMY IN DIFFERENT EDUCATIONAL CONTEXTS IN CATALONIA
}

\begin{abstract}
New Public Management (NPM) emerged in the political debate since the eighties, with the promise of improving the effectiveness of the public sector, through the modus operandi of private enterprise. School autonomy is a key policy in the agenda of NPM. The aim of this research is to analyze the recontextualization of school autonomy in public schools in Catalonia. This investigation is based on the hypothesis that the social composition and stability of the teaching staff influence the recontextualization of school autonomy in schools. The methodology is based on a comparative case study. Two public primary schools with different school contexts have been selected. The case study combines surveys with school staff, semi-structured interviews with key stakeholders and direct observation. The results show that the school context can trigger different responses and limit the development of pedagogical autonomy in disadvantaged environments.
\end{abstract}

Keywords

New Public Management; School autonomy; Recontextualization; Endogenous privatisation

NOVA GESTÃO PÚBLICA E REFORMA DA EDUCAÇÃO: RECONTEXTUALIZAÇÃO DA AUTONOMIA DAS ESCOLAS EM DIFERENTES CONTEXTOS ESCOLARES NA CATALUNHA

Resumo

A Nova Gestão Pública (NGP) surgiu no debate político a partir dos anos oitenta, com a promessa de melhorar a eficácia do setor público através do modus operandi da empresa privada. A autonomia escolar é uma política 
essencial na agenda da NGP. Esta pesquisa analisa a recontextualização da autonomia escolar na escola pública na Catalunha. A hipótese é que a composição social e a estabilidade do corpo docente influenciam a recontextualização da autonomia escolar. A metodologia usada é fundamentada no estudo de caso de tipo comparativo. Duas escolas primárias públicas com diferentes contextos escolares foram selecionadas. $O$ estudo de caso combina pesquisas com professores das escolas, entrevistas semiestruturadas com os principais personagens-chave e observação direta. Os resultados mostram que o contexto escolar pode desencadear respostas diferentes e limitar o desenvolvimento da autonomia pedagógica em contextos desfavorecidos.

\section{Palavras-chave}

Nova Gestão Pública; Autonomia escolar; Recontextualização; Privatização endógena

Toda a correspondência relativa a este artigo deve ser enviada para: Lluís Parcerisa Marmi, Universitat Autònoma de Barcelona, Departament of Sociology, Edifici B - Campus Bellaterra, 08193 Bellaterra (Barcelona), España. E-mail: Iluis.parcerisa@uab.cat 